(C) [2008] IEEE. Reprinted, with permission, from [Zenon Chaczko, Ryszard Klempous, Jan Nikodem and Jan Szymanski, Applications of Cooperative WSN in Homecare Systems, 2008, Third International Conference on Broadband Communications, Information Technology \& Biomedical Applications, 2008]. This material is posted here with permission of the IEEE. Such ermission of the IEEE does not in any way imply IEEE endorsement of any of the University of Technology, Sydney's products or services.Internal or personal use of this material is permitted.

However, permission to reprint/republish this material for advertising or promotional purposes or for creating new collective works for resale or redistribution must be obtained from the IEEE by writing to pubs-permissions@ieee.org. By choosing to view this document, you agree to all provisions of the copyright laws protecting it 


\title{
Applications of Cooperative WSN in Homecare Systems
}

\author{
Zenon Chaczko $^{1}$, Ryszard Klempous ${ }^{2}$, Jan Nikodem ${ }^{2}$, Jan Szymanski ${ }^{1}$ \\ ${ }^{1}$ University of Technology Sydney, NSW, Australia \\ ${ }^{2}$ Wroclaw University of Technology, Poland \\ E-mails: zenon.chaczko@uts.edu.au, ryszard.klempous@pwr.wroc.pl, \\ jan.nikodem@pwr.wroc.pl,jan.szymanski@uts.edu.au
}

\begin{abstract}
Cooperation plays the crucial role in shared space of the homecare processes. It is a rather hard task to ensure effective cooperation in home care environment. This is due to variability of schedules, tasks and mobility of both patients and carers. In this paper, we discuss sensor network technology that can facilitate and improve home care cooperation scenarios. We present methodology, recommendations and applications for incorporating a WSN based solution in various areas of Homecare. We argue that even the most difficult areas of cooperation between patients and their carers such as: information retrieval, information dissemination, scheduling, coordination of short and long-term treatment can be supported by WSN based solutions. Finally, we discuss sensor network design approaches for incorporating smart communication devices and sensors to support health care workers and their patients in their daily activities. The network of smart sensors can help to maintain awareness of the activities of all stakeholders and the need to integrate communication and computer technology with the requirements of effective aged care infrastructure.
\end{abstract}

Keywords: Homecare systems, WSN, resource constrained systems

\section{INTRODUCTION}

Humans biologically age not just as individuals or selected communities in the industrialised countries but the world population as a whole. According to recent statistics [10], in 2006, almost 0.5 billion people worldwide were 65 years and older. Older persons (persons 65 years and older) in Japan, Australia, and Europe amount almost one fifth of the total population (NIPSS 2008). Similar trends can be observed in U.S., where the older part of the population represents 37.3 million people ( $1 / 8$ of the total American population in 2006). In twenty years (2030), in US alone, there will be approximately 71.5 million aged persons (twice their number in 2000). In 2030, only the $85+$ segment of population alone is estimated to reach around 9 million. Also, by the same year, the total number of older persons around the world is projected to increase to almost 1 billion, approximately, 1 in every 8 of the earth's inhabitants. Most noticeable, the fastest increases in the aged population are occurring in developing countries, as these will witness a rapid increase of 140 percent by 2030. Majority of elderly persons suffer at least one chronic condition and many have multiple illhealth conditions. The most frequently occurring conditions among aged people (2003 statistics) are: hypertension (51\%), diagnosed arthritis (48\%), range of heart disease $(31 \%)$, various cases of cancer $(21 \%)$, diabetes (16.0), and sinusitis (14\%). Despite of these facts a relatively small percentage ( $4.5 \%$ on average) of the $65+$ population lives in nursing homes. The percentage increases dramatically with age, ranging from around $1.1 \%$ for persons $65-74$ years to $18.2 \%$ for persons $85+$. In coming years living arrangements of aged people are unlikely to undergo dramatic changes as most of elderly have a preference to stay at their homes as long as it is only possible. However, in most countries, the number of aged persons living alone is dramatically increasing. Following the latest US statistics, around 30 percent ( 10.0 million) noninstitutionalised older persons live alone in their households see the Figure 2. In some European countries, around $40 \%$ of women at the age of 65 and more live alone. Similar situation takes place in societies with established traditions of older parents living with children (i.e. Japan or China) where traditional living arrangements are becoming less common (see Fig. 3).

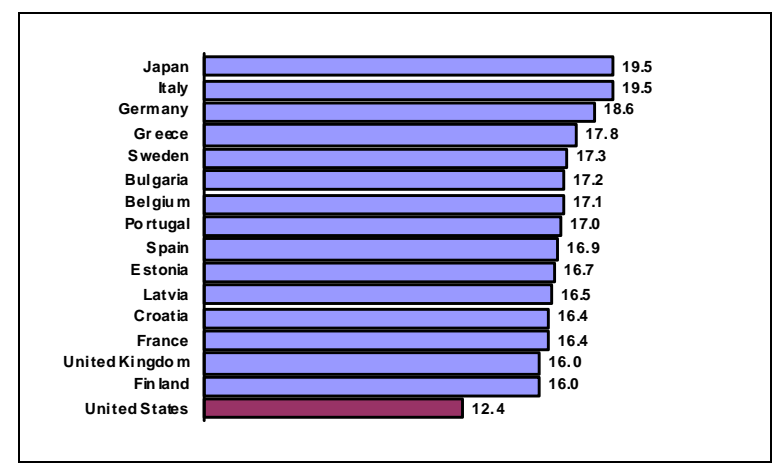

Fig. 1: Aging Population around the world. Source: Haub (2006) 


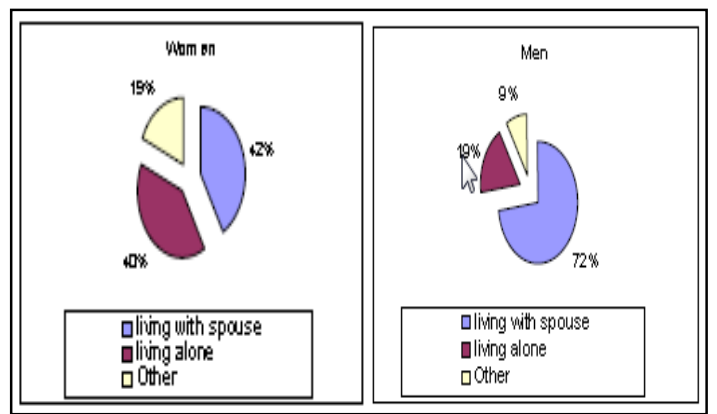

Fig. 2: Aging Population and living arrangements in US. Source: USDHHS (2008)

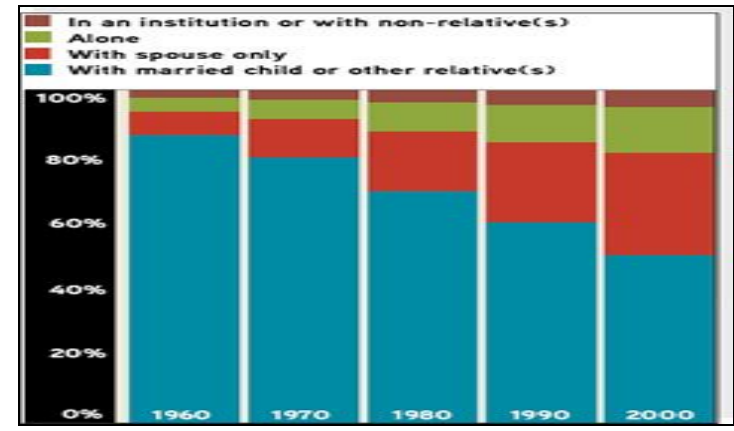

Fig. 3: Aging Population and living arrangements in Japan. Source: (NIPSS 2008).

In many countries and communities, the most serious strain on societal, institutional and economic capacity for support is going to be related to a rapidly increasing number of the aged persons in $85+$ years bracket.

\subsection{Rationale}

Traditional delivery of medical care in majority of cases involves scheduled (planned) and unscheduled patient's consultations, medical check-ups and administration of medicine. Health check-ups usually involve routine examination tasks commonly representing just a temporal snap-shot of a person's general psychological and physical condition (Warner 1996). Extensive and intensive examination often involving continuous gathering of the medical data and then a laborious, processes of data analysis can be slow, time consuming and expensive. The quality of healthcare delivery process can be significantly improved if frequent, timely and accurate patient health history is being kept and properly (regularly) analysed (Campbell 1998), (Fitzpatrick 2000). Frequently, aged persons with a chronic condition(s) have to remain at a medical or palliative care facility for a purpose of monitoring of their general well-being, or routine tests after an operation (or even minor medical treatment). Significant medical resources could be saved if aged/sick persons or their carers could perform general and routine health checks themselves at their homes, providing access to required facilities is made available and adequate procedures are followed. In many cases it would be not only feasible but representing a best option for the patient. With support of WSN technology, the quality of medical support could be even improved through regular and frequent (continuous) monitoring.

\subsection{Aims and Objectives}

The research initiative focuses on application of innovative hardware and software applications while exploiting newly developed WSN concepts, cooperative agent computing paradigms and algorithms (Krishnamurthy et al. 2006), (Kennedy, 2006). The main goal of our study is to investigate privacy non-invasive, safe, secure, reliable, autonomous agent based solutions for monitoring health and activities of elderly or/and sick persons at their homes. The proposed solution involves the development of two major components:

- A generic hardware platform based on UC smart sensor technology. The hardware can be used as a wearable device or as a easily installable device in a variety of infrastructure fixtures (i.e. power points, lighting fittings) or domestic appliances.

- Middleware framework for rapid application deployment of WSN based homecare systems at family homes, healthcare centers or other facilities.

\section{COOPERATIVE HOMECARE SYSTEMS}

In delivery of effective computerised healthcare system for the aged persons, cooperation in a shared space of various components and participants plays the fundamental role (Dourish \& Bellotti 1992). Patients may be provided with services and care from a range of different stakeholders (i.e. healthcare workers, family members, friends, neighbours and the community). Depending on various arrangements, care oriented computer and embedded devices as well as sensors may assist humans in direct cooperation tasks and in the ambient manner (e.g. facilitating enquiries, phone calls, recordings logs or teleconferences, generating data for ad-hoc charting, etc.). While working in a continuous mode, various embedded devices and sensors can facilitate cooperation processes by recording, fusing and providing support for keeping the medical records and data analysis of patient health (see Fig 4). Pervasive access to information allows participants to share a rich set of data about patients and their environment. Various life threatening and health problems can be possibly identified and resolved early as treatments for various ailments can be timely and accurately coordinated and executed. In home care environment, a human medical support is often mobile and only infrequently may have a chance to see each other inperson. In practice, the homecare medical staff delivers service care to patients at their homes and spending limited time accessing computers while at work. The difficulties with cooperative information sharing or exchange force medical support staff to make ad-hoc decisions and carry out medical treatments without prior 


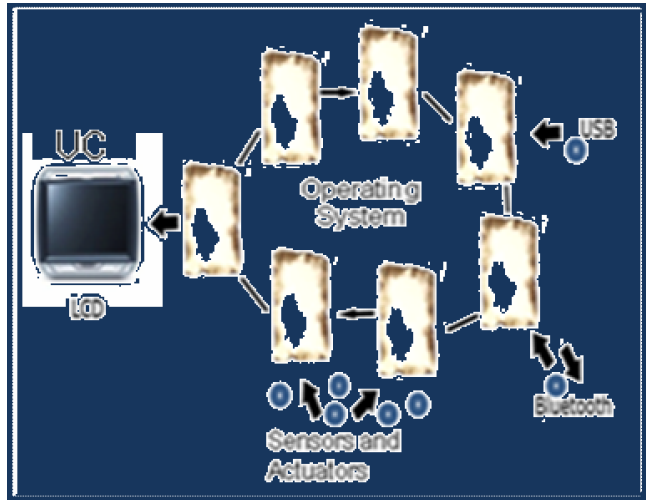

Fig. 4: Operating principles of the homecare system access to often critical information about patients that otherwise would be easily available. Networks made of communication, computing devices and sensors (actuators, wearable computing devices) WSNs are capable to sense, observe, process, react, obtain and deliver a broad range of temporal, spatial, physical, biological, medical and social data about patients. WSN applications can continuously generate large amounts of real time, monitoring data that can both be processed at the node or/and transferred to computer network via interconnected WSN infrastructure. With rapid advances in electronics, sensor and computing technologies it is possible to build very powerful dynamic and wireless communication network infrastructures. Such infrastructures are capable to facilitate extraction, delivery, access and mapping of spatio-temporal and behavioural data in an ambient manner to various users.

\section{THE UNIVERSAL COMMUNICATOR}

The Universal Communicator (UC) is a small electronic wearable device that is equipped with a miniature display that allows not only a display of pre-stored, collected or processed data but can also assist communication and control of various computerised devices available in the environment. Although the UC can function as a standalone device it can be an integral part of a much larger system i.e. a smart home or a smart hospital. Thanks to its wireless communication capability the UC can receive vital information about the environment (i.e. presence of dangerous gases, chemicals or radiation) and present it to its users see Fig. 5. With a speaker, microphone and connectivity to variety of sensors (including health monitoring devices) the device can facilitate the delivery of effective computerised healthcare system for the aged persons. With the GPS, RFIDs and accelerometers together with localisation algorithms the device can be used not only for monitoring events but to accurately locate people and the equipment in the selected, dynamic environment.

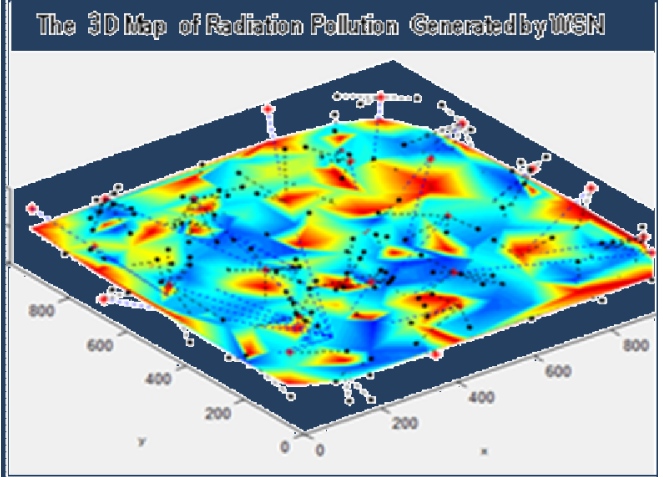

Fig. 5: Operating principles of the homecare system

Immediate applications would allow the adaptation of the device to control working/living environment to the specific needs of the user (i.e. regulation of temperature, lighting or humidity). Other applications could possibly involve support for users moving in an unknown environment, elderly or blind persons. We are working on applications that involve monitoring of various aspects of human health and performance of humans when playing sports. The UC can be directly equipped with heart-bit-rate, blood pressure, perspiration, temperature and many other sensors including EKG and EEG. If required, wireless connectivity would allow for the constant flow of data to medical personnel.

\section{DESIGN AND PROGRAMMING OF THE UC}

The UC with its dimensions (i.e., 52mm length, $40 \mathrm{~mm}$ width and 2-7 $\mathrm{mm}$ thickness) resembles the size of a standard badge that people usually wear at work or at various functions. In its basic version the UC is equipped with a 16-bit PIC24FJ64 processor that is capable to perform, $16 \mathrm{mln}$ operations per second, 64 Kbyte internal memory, 8 Kbyte RAM, 2 UART (Universal Asynchronous Receiver Transmitter) and 2 I2C (Inter-Integrated Circuit) ports. The processor is small, relatively simple and inexpensive. It allows easy programming and connectivity of the UC with other devices. The basic UC has a colour 1.5 inch LCD (OLED technology) screen with capability to display $128 \times 128$ pixels in 65000 colours. This allows for a decent resolution of a text, moving pictures, graphs, maps, etc. The mini USB port connection allows for a direct connection with a PC if the direct data transfer is required. The SPI (Serial Peripheral Interface) port, allows for easy download of a new software. The Flash memory, typically 512 Kbyte ensures there is enough capacity to store data required data and it can be extended further by $64 \mathrm{MB}$ of mini-SD card reader. The Bluetooth interface allows the UC for wireless communication. Several UCs can form a wireless 
network (WSN). The device has a $3.7 \mathrm{~V}$ rechargeable battery.

In the past, there were several projects which involved similar to the UC wearable devices. For example, projects that involved smart communication devices (smart badge) were described in by Locher (2002) and White (2006). White, in his smart badge project, used RF and IR communication methods to ensure external connectivity and his devices used the CC1010 microcontroller (compatible with 8051). Locher, however, used AVR ATMEGA128 controller applying the standard Bluetooth (BT) interface for communication with external devices. In our project, we also used the BT based wireless communication. Our decision was based on a basis of the better performance of the BT technology if compared with traditional RF/IR. Additionally, we could use of-the-shelf standard BT APIs for data transmission while other solutions would require additional programming efforts. For the initial version of the UC device we have used the PIC24 (Programmable Interface Controller) as depicted in the Fig. 5. The newer version of the UC uses a touch screen (Fig. 6) to allow for better control and communication with various devices in the environment. In works by Huebscher and McCann (2004), Fahy and Clarke (2004), Boselli et al. (2005) the authors have proposed their own multilayered software system solutions that were somehow adjusted to the specifics of the architectures of their chosen microcontroller devices. Similarly, in our project the choice of PIC24 controller has determined the initial architecture of the proposed UC software system.

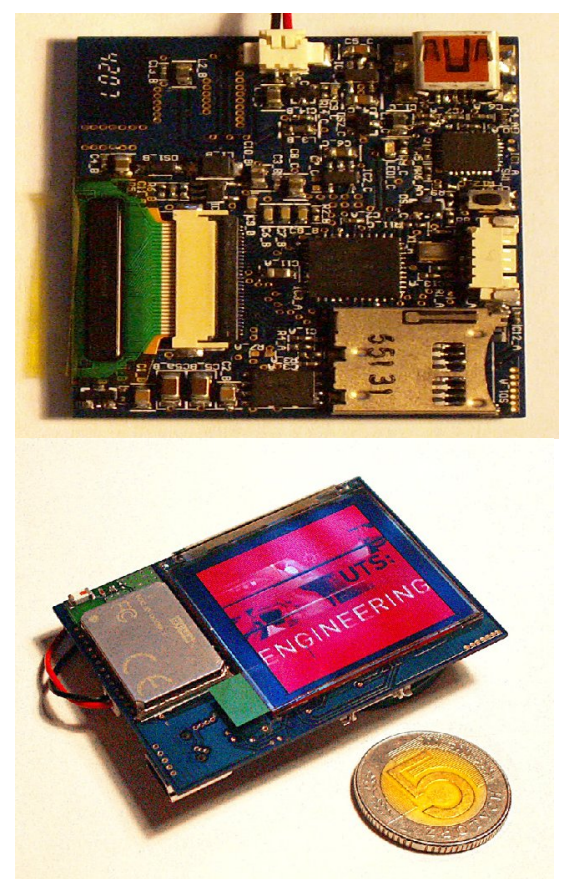

Fig. 5 Universal Communicator, initial version of the (PIC24 based) device.

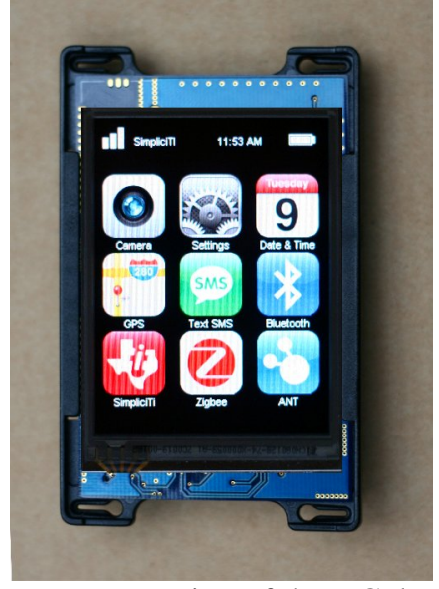

Figure 6: Latest version of the UC device.

The UC based system uses a software development framework based on the MPLAB environment. The main task of the framework is to allow proper configuration of the equipment (i.e. the microprocessor, input/output ports, interrupts services, USB Bluetooth drivers and its sensors). The drivers are serviced using the UART channels and the SPI ports is used for the upload of software or its upgrade. A general operation of the software system is shown in Fig 4. The content of 64 Kbytes of the Flash memory can be cyclically displayed on the LCD screen. The content can be upgrade at anytime using the Bluetooth or the USB ports. The UC can display graphically various environmental data received from the attached sensors, sensors that are embedded in the environment or sensors from other UCs in the neighbourhood. The data can be passed to other computer systems that can play a supervisory role.

\section{EXPERIMENTATION}

Experiments and various simulation tests have been conducted using the WSN-based systems in which a newly developed communication device called Universal Communicator was able to display various data, graphs and maps generated both by both real and simulated wireless sensor devices in homecare system environment. Also, a range of simulation tests were performed to study scenarios and mechanisms of UC self-organisation, self-configuration and sensor localisation. The Figures 7,8 and 9 demonstrate the experimental mappings of cooperating sensors that can be displayed on the UC screen. These mappings can include associations between UC and sensors, sensor and UCs positions, their clustering and energy levels of sensor batteries in the homecare environment. 


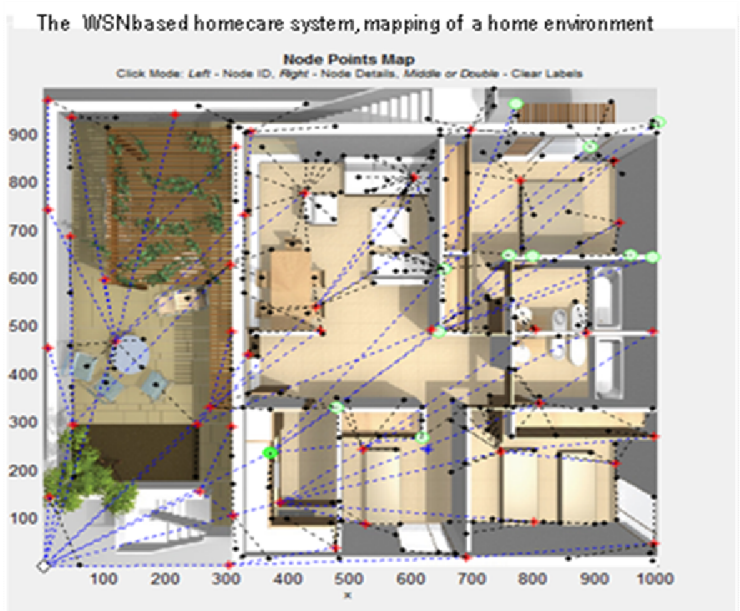

Fig. 7: The UC display of cooperating sensors in the homecare environment

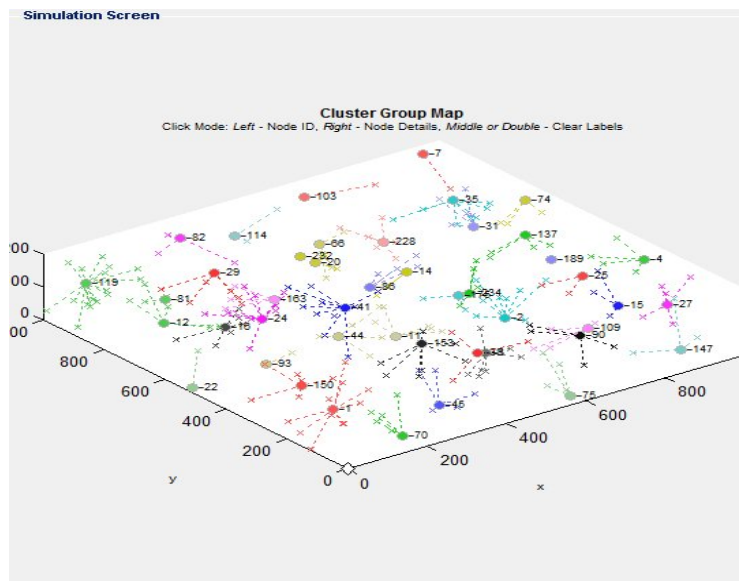

Fig. 8: The UC display of the map of cooperating clusters in the homecare system

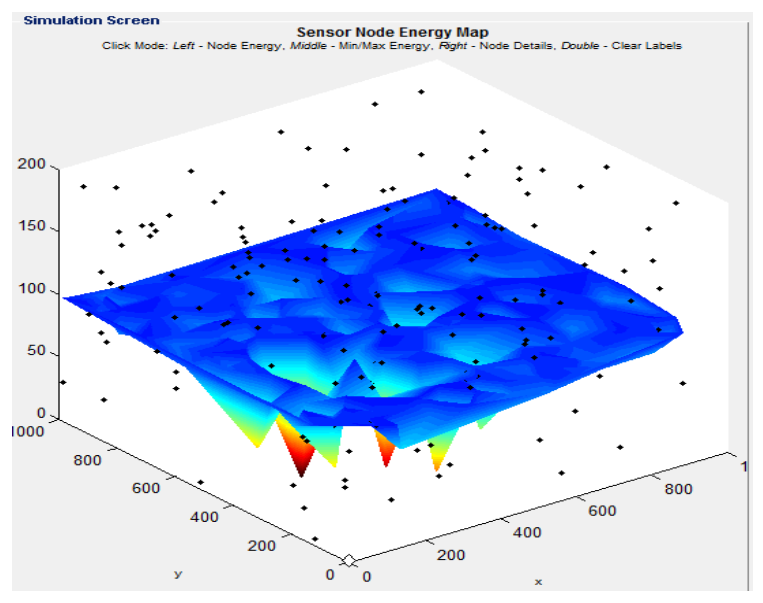

Fig. 9: The UC display of the energy map of cooperating sensors in the homecare system

\section{FUTURE DIRECTIONS}

Reliable, portable and easily configurable network management services (software components) and low- cost UC devices have important roles to play in the architectural design of homecare systems. Softwarebased and resource constrained UCs can be easily deployed and incorporated into any existent home infrastructure (i.e. internet, power points, light fittings, gas/electricity/water control gauges, alarm devices, door locks, etc.) and any set of appliances. Ultimately our work on UC sensors and applications would lead us to design and development of inexpensive and reliable homecare systems. There are many technical challenges and obstacles that need to be addressed to realise the vision of open, sensor-based, interconnected with robotic and computing devices, infrastructures that could allow development of new generation of homecare applications. Future investigations will have to address technical questions how to build and interconnect infrastructures that use heterogeneous sensors and computer devices as well as how to seamlessly discover and access them. Future studies will also aims at answering questions on how to fuse, format and contextualise sensor data. There are challenges that are pertinent to the human psychology and fallacies related to application of WSNs that have grown in recent years. These fallacies often relate to issues of cooperation, transparency, the balance between autonomics and the central control; as well as human issues of ethics, privacy and personal freedom that remain at the core of the real progress in the development of the future homecare systems.

\section{CONCLUSION}

In this initial study, we are focusing on the phenomenon of cooperation in homecare systems. The homecare cooperation is constrained by characteristics of the specific settings, including the mobility of healthcare workers, clinicians, schedule variability of team members, and the number of face-to-face meetings between team members. These difficulties can be partially addressed by ICT technologies including pagers, the voice mail, cellular phones and similar devices. However, these traditional technologies are still inadequate in addressing some of the complexities of collaborative activities that clinicians would like to engage in as a part of the care delivery. Homecare clinicians require a support for scheduling visits, disseminating of information, retrieving information from others, coordinating treatments, and creating care plans. Mechanisms based on the traditional ICT technologies allow for a flexible and ad-hoc sharing of patient records help to address these needs partially only. By integrating a more complete cooperation support that exploits the complex WSN-based system infrastructure (Newman 2004) we are able to establish an integrated communication and situation/context 
awareness in a non-invasive manner. This new approach could significantly enhance the quality of homecare environment. The key innovation is in applying the "hardware-in-the-loop" thinking when designing software systems that use the WSN. At the very beginning of the project, we directly put not only the purpose and scope of the mission but tasks, resources and human activities in mind as well. At present, we are not aware of any specific commercial technology in the market that could help to effectively solve the problem we are aiming at. The marriage of sensor networks and standard appliances technologies should be able to release people from some of the laborious and mundane tasks; and possibly reduce the stress frequently associated with the healthcare work. In this paper we discuss suitability of the new generation of computing devices (UC) and software solutions that we have programmed and tested for applications in homecare systems. This research is a part of an ongoing project with homecare systems conducted at UTS and WUT. Future work will include field-based investigations that expand on these findings.

\section{REFERENCES}

Boselli, R.; Cabitza, F.; De Paoli, F.; Loregian, M. (2005) An Adaptive Middleware to Support Context-aware Knowledge Sharing, 25th IEEE International Conference on Distributed Computing Systems, 6-10 June, Page(s): 352 358, 2005.

Campbell, J.R, (1998) Strategies for problem list implementation in a complex clinical enterprise. Proc. AMIA Symp.

Fahy, P. and Clarke, S. (2004) CASS - a Middleware for Mobile Context-aware Applications, Workshop on Context Awareness, MobiSys

Huebscher M., McCann J. (2004) Adaptive Middleware for Context Aware Applications In Smart-Homes, 2nd International Workshop on Middleware for Pervasive and Ad-Hoc Computing, Middleware 2004, Canada, October,

Locher I. (2002) Design and Implementation of iBadge and its Distributed Speech Processing Capability, University of California, Los Angeles,

Dourish P. and Bellotti V. (1992) Awareness and Coordination in Shared Workspaces. Proc. Computer Supporter Cooperative Work (CSCW'92), ACM Press, p. 107-114.

Kennedy, J. (2006) Swarm Intelligence, in Handbook of Nature-Inspired \& Innovative Computing, Ed: A. Zomaya, Springer Verlag, New York, 187-221.

Krishnamurthy, E.V. \& Murthy, V. K., (2006) Distributed Agent Paradigm for soft and hard computation, Journal of Network and Computer Applications, Vol. 29, 124-146.
Newman, M.E.J., (2004) The Structure and Function of Complex Networks, Santa Fe Institute Publication.

Fitzpatrick G. (2000) Understanding the paper health record in practice: implications for EHR's. CD Proc. Health Informatics Conference (HIC2000), Adelaide, Australia.

Haub C. (2006) World Population Data Sheet, National Institute of Population and Social Security Research. Population Statistics of Japan 2003. Available at: http://www.ipss.go.jp/index-e.html. Accessed January 28.01.2008

USDHHS (2008) US Department of Health and Human Services report, Accessed 12.01.2008, http://www.aoa.gov/prof/Statistics/statistics.asp

White M. (2006) SmartBadge:An Electronic Conference Badge using $R F$ and IR Communications, Master of Engineering Thesis, University of Canterbury, Christchurch, New Zealand,

Warner I. (1996) Introduction to telehealth home care. Home Healthcare Nurse 1996; 14(10):790-6. 